\title{
Cryptography Policy-Based Data Communication in Trusted Environment
}

\author{
Rajkumar N. ${ }^{1}$, Janhavi V. ${ }^{2}$ and A.B. Rajendra ${ }^{3, *}$ \\ ${ }^{1}$ Accendere Knowledge Management Service Pvt Ltd, New Delhi, India \\ ${ }^{2}$ Department of Computer Science and Engineering, Vidyavardhaka College of Engineering, \\ Mysuru, Karnataka, India \\ ${ }^{3}$ Department of Information Science and Engineering, Vidyavardhaka College of Engineering, \\ Mysuru, Karnataka, India; abrajendra@vvce.ac.in
}

\begin{abstract}
Objective: This study analyses out of exchanging security information. There is a strong requirement for access to various concentrations of delicate information at the edge; however, there is a greater danger than the key business setting at that place. Methods: This study explores the demands, technologies, and methods of threat mitigation to safely share information with the tactical user while preserving data and information systems from intruders and malware. Findings/ application: The new cryptography policy architecture requires to eliminate the architecture of stovepipe and free the accesses to communicate data across traditional and non-traditional field limits.
\end{abstract}

Keywords: Cryptography, Encryption, Decryption, Trust

\section{Introduction}

Many developing applications will depend on comprehensive shared collaboration between a computer network that is extremely interconnected. 1,2 Depending on climate forecasts acquired directly from the computers of the local climate station, an enclave of computers working together may aim to put up thermostats. The car that communicates to adjacent cars, computers may choose the greatest way of action to avoid an imminent crash. Sensors tracking a person's essential inner body features on the street can transmit premature alert indications to the closest clinic via multihop Adhoc networks to enable prompt reactions.

In such applications, it is anticipated that each system will fulfill some functions for the network's general benefit. In such situations, an apparent necessity is the capacity to control the equipment. Realizing extensive acceptance of such applications requires computers that are adequately trustworthy to be realized at a small expense. With the realistic applications deployed, it can also address several security problems impacting the existing network infrastructure through the ability to build trustworthy low-consumer computers. Trustworthy

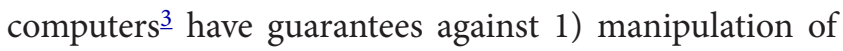
data by such programs and 2) exposure of the specifics used to authenticate applications. ${ }^{4}$

The author introduces the RT system, a compilation of role-based trust-management dialects, to describe techniques and skills in distributed acceptance. RT combines the advantages of role-based access control and trust management systems and is particularly appropriate for attribute-based access control. They introduced four parts of the RT structure: RT1, RT2, RT T, and RT D. They have seven types of credentials together and promote localized functions power, position description delegate, related functions, parameterized positions, multiple functions, and task activation assignment. $\underline{5}$

This research provides a model for a secure rolebased communication system oriented on X.509 position allocation records and the role-based permission system guided by the PERMIS strategy. The proposed design

${ }^{*}$ Author for correspondence 
was created in an attempt to make a few changes as possible to current email systems and protocol norms. The assumption is that such a design will make it easier for companies to deploy. They have two layout variants to send digitally signed role-based posts. They are presently constructing both systems and will inform in owing time of execution, efficiency, and usability. $\underline{6}$

The author developed D1LP to explain security policies and certificates of authorisation across large, transparent, and decentralized networks. Delegation Logic (DL) is the monotonic variant of the trust-maker language based on logic. They included a list of template policies for assessing the expressive capacities of TM systems and found there were no major expressive capacities in previous TM systems. Such structures, for example, are exceptions. Policymaker, which allows programs to be used in general programming languages in credentials and techniques and therefore can express almost any strategy. In short, the major contribution made in this article is the TM D1LP language which is concise, stated, tractable, and realistic. Researchers also studied how delegations of a wide and limitless scope, delegations to various major buildings and delegations were sponsored and some of these functions are important. $\underline{\underline{Z}}$

A technique of ensuring a wireless network while offering significant entry for guests. An extra permission test depending on SPKI/SDSI records was introduced to the EAP-TLS authentication phase. - We eliminate the need for a cumbersome key agency by using SPKI/SDSI; by greasing it on the bottom of the existing X.509-based PKI, we do not allow our customers to purchase extra client software. Our objective is to develop a method that performs delegation in a manner that represents real-world permission stream that does not depend too strongly on a distributed power; SPKI/SDSI enables us to achieve this objective. Our potential research will enable us to explore how our alternative matches with other current concepts, which will hopefully lead to a remedy that is safe, fully decentralized and able to adapt to fresh technologies and delegate strategies. $\underline{9}$

A policy that uses the methods of computational thinking to suggest such semantic mappings. Our strategy is focused on well-founded concepts of semantic similarity, described in aspects of the shared allocation of probability of the ideas concerned. We defined the use of machine learning, and especially multi-strategy training, for differences in the computing notion. This teaching method allows our strategy to be readily extended to extra learners and thus to exploit extra types of instance information. Finally, we brought relief branding to the ontology-matching framework and demonstrated that it can be tailored to effectively leverage a range of heuristic expertise and domain-specific limitations to further enhance fitting precision. Our tests have shown that on several real-world fields we can fit $66-97 \%$ of the nodes correctly. $\underline{10}$

Every system is regarded as a reliable computer in the event of the current system. And so the intruder discovers it simple with false messages to assault the system. And also where many are used for some useful intent in the evolving network. And there's a ton of opportunity to transmit unwanted data to the enemy in those. In the event of the fire alarm, they could give false alarm where it leads to a high drop if all the device is regarded as safe. And so to safeguard it, we need a system. This is why we are developing a fresh system.

\section{Proposed Methodology}

We are introducing a fresh technique for the proposed system to maintain the network. The previous route is accomplished. Realizing extensive acceptance of such application machine instructions that are adequately trustworthy to be realized at small expense. In addition to allowing the implementation of realistic applications, many of the security issues threatening our existing network infrastructure are addressed through the ability to create credible low cost computers. While "cheap" and "trustworthy" might at first glance seem to exclude each other, a viable approach is to reduce living within the safe confines of the parts. It is far from dogmatic that the often found assertion that "complexity is the enemy of security". For one, reduced complexity means stronger enforcement verifiability. Furthermore, maintaining the difficulty at small concentrations within the confidence limit can eliminate the need for proactive heat dissipation interventions. Restricted strategies to enable protection concurrently and heat dissipation appear to be costly. On the other side, it can be safe and cheap to promote unconstrained protection policies.

\section{Design and Working Principle}

First, the User registers his account by providing his login credentials consisting of Name, Username, Password, 
and Email ID. The client will provide the appropriate username and password and then login.

Any reliable computer establishes a trust border to crystallize. For example, all chip elements will dip into a single ScP chip within that trust limit. Proactive steps for the safety of components within the boundaries are the implementation of the trust limit. However, the areas within a physically secured trust boundary may dynamically change based on the $\mathrm{ScP}$ state. When the CPU is off, security cannot be extended to all areas. However, when the CPU is operated, the scope of security must be broader. The user reaches and uploads the document into the protected area. The Non Trusted Party cannot enter the trusted region or the files in the trusted region cannot be accessed. After this method is completed, they are shifted where the encryption and decryption occurs.

Next, we investigate DOWN's suitability for Identitybased encryption (IBE) and identity-based signature (IBS) systems. We then need an ID-based identification system of small difficulty for ScPs for changing situations of applications. This involves an outline of some current ID-based KPS with low complexity.

For decryption and signing, the private exponent $d$ is used. More specifically, to perform computations such as decryption and marking, the individual exponent must be placed in RAM. The square-and-multiply algorithm is often used to perform a modular exponentiation.

The encrypted file is sent with the key to the untrusted system, normal file is sent to the trusted system and only files are read when the files are sent and the file path is stored in the database. Before moving the mail to reliable and untrusted devices, we must ensure that the system is running so that the customer can obtain documents. Encryption is performed for privacy purposes. The documents are encrypted because it is an untrusted system. Encryption transforms the simple code into code. It is impossible for people to access this cipher text. This is to keep privacy so that the untrusted system does not access the documents.

Then it receives the files. If it is a trusted system, the files receive in encryption mode with a secret key to decrypt the encryption file and view the file without decryption. Usually the documents are placed in "c: $\mid$ receive" route. The user cannot alter or alter the file if it is a read-only file. Encrypted files must be transformed to simple document in code mode to be accessed. So you have to decrypt the files. Decryption is the plain text transformation cipher text so that it can be accessed as shown in Figure 1.

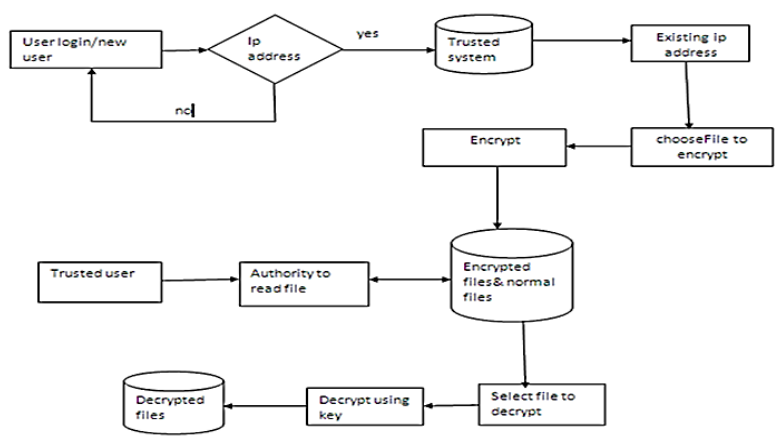

Figure 1. Working principle of cryptography policy-based data communication in trusted environment.

\section{Results}

The method that has trusted and untrusted two systems. Some security policy was defined by a trusted system in the safety technique. System audit traces all software calls to the device. Untrusted scheme auditing is enabled as such, a reliable scheme may violate a defined safety policy. As it becomes more desirable to use and share data among applications, we have seen the downside of delicate data being available to organisations for which it was not designed. There is no link to authorized sides in this untrusted system. .11 The untrusted group is unable to obtain authorized group files. Key authentication is a popular characteristic of procedures that use end-to-end authentication that can be downloaded by the authorized group through confidential main checking and records can be modified. The untrusted person can download only, but cannot create changes.

\section{Conclusion}

This study's encryption policy significantly reduces the circuitry structure needed for countermeasures and increases the guarantees provided to secure privacy by reliable devices, such as cryptographic co-processors. This is done by eliminating reliance on costly and sensitive multi-stage countermeasures. Therefore, cryptography laws can simultaneously reduce and improve the accuracy of cryptographic co-processor prices. To accomplish this, cryptography needs the ability to execute calculations with parts of fractional myths. Many asymmetric cryptographic primitives have been shown to contribute rapidly to cryptography. Specifically, asymmetric schemes that use concealed switches for numbers are restricted to 
computations, and exponentiation rapidly lends itself. The encrypted keys of IBE schemes or systems for which standard multiply reverse calculations include personal key operations are currently not easily secured, it would appear. Furthermore, schemes for which there are no limitations on the choice of concealed switches are chosen for use in conjunction with cryptography.

\section{References}

1. Ramkumar M. Trustworthy computing under resource constraints with the DOWN policy. IEEE Trans Depen Secure Comp. 2008;1(1):49-61.

2. Kwiatkowska M, Sassone V. Science for global ubiquitous computing. In: Hoare T, Milner R, editors. Grand challenges in computing (research); 2004. P. 1-9.

3. Smith SW. Trusted computing platforms: design and applications. Springer; 2005.

4. Anderson R, Bond M, Clulow J, Skorobogatov S. Cryptographic processors-a survey. computer laboratory technical report UCAM-CL-TR-641. University of Cambridge; 2006. vol. 94(2). P. 357-69.

5. Li N, Mitchell JC, Winsborough WH. Design of a rolebased trust-management framework. In: Proceedings IEEE symposium on security and privacy; 2002. P. 114-30.

6. Chadwick D, Lunt G., Zhao G. Secure role based messaging. Int Fed Inf Process. 2005;175:263-75.

7. Li N, Grosof BN, Feigenbaum J. Delegation logic: a logicbased approach to distributed authorization. ACM Trans Inf Sys Secur. 2003;6:1-44.

8. Goffee NC, Kim SH, Smith S, Zhao M, Marchesini J. Greenpass: decentralized, PKI-based authorization for wireless LANs. In: 3rd annual PKI research and development workshop proceedings; 2004. P. 1-16.

9. Smith S, Goffee NC, Kim SH, Zhao M, Marchesini J. Greenpass: flexible and scalable authorization for wireless networks; 2004. P. 1-20.

10. Doan A, Madhavan J, Domingos P, Halevy A. Learning to map between ontologies on the semantic web. In: WWW; 2002. P. 1-12.

11. Trusted systems: protecting sensitive information. [cited 2018]. https://www.123helpme.com/view.asp?id=22290. 